\title{
Étude de l'effet d'échelle de pieux battus en cours de centrifugation
}

J.-G. SIEFFERT

École Centrale de Nantes

1, rue de la Noë

44072 Nantes Cedex 03

D. LEVACHER

Université du Havre Quai Frissard, BP 265 76055 Le Havre

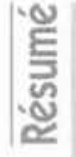

L'objectif de cette contribution est de faire le point sur la validité des lois de la similitude dans le cas du battage en cours de centrifugation. Deux sêries d'essais ont été réalisées dans des conditions très particulières. Dans le premier cas, le même pieu-modèle a été soumis à trois niveaux d'accélération: les trois essais correspondent donc à trois pieux-prototypes différents. Dans le deuxième cas, on a utilisé quatre pieux-modèles de dimensions proportionnelles, et respectivement soumis à des niveaux d'accélération tels qu'ils représentent tous les quatre le même pieu-prototype. Dans les deux cas, on montre que les lois de similitude sont globalement vérifiées, ce qui justifie le développement des études en centrifugeuse sur le comportement des pieux battus.

\section{Scale effect analysis of piles driven in centrifuge}

In orcier to achieve correct scaling of the pile driving in centrifuge, the inherent similitude laws must be properiy checked. Two series of model tests were conducted with their specific conditions. In the first series, the same model pile has been impacted at three different acceleration levels. Thus, the resulting three tests are representative of three different prototype piles. The second series was undertaken to study the model of modelling. Four model piles - proportionaly dimensioned - and tested at the respective acceleration level such as, together, they simulate the same prototype pile. In both cases, conclusions demonstrate that the similitude laws applied to the pile driving are globally checked, so this justifies the investigation in a wide variety of driven pile problems. 


\section{PRINCIPALES NOTATIONS}

$\sigma \quad$ : contrainte

x : coordonnée

u : déplacement

p : : masse volumique

g : accélération de la pesanteur

t : : temps

E : énergie

$\mathrm{H} \quad$ : hauteur de chute du marteau

$\checkmark \quad$ : vitesse particulaire

$F \quad$ : force d'impact

$\mathrm{S}$ : section

M : masse

$\mathrm{z}$ : impédance

$\mathrm{N}^{\alpha} \quad$ : facteur d'échelle

a : accélération

ф. : diamètre

c : vitesse de propagation des ondes

n : nombre de coups

f : : cadence de battage

$\bar{E} \quad$ : module d'élasticité

$\tau_{\mathrm{m}} \quad$ : durée d'un aller-retour dans le marteau

$\tau_{\mathrm{p}} \quad$ : durée d'un aller-retour dans le pieu

$\tau$ : durée du choc réel

$\tau_{0} \quad$ : durée du choc théorique

$\theta \quad$ : durée du palier de contrainte maximale

L. : longueur de l'ébranlement

$1_{1}, 1_{2} \quad$ : position des jauges

$\delta$ : enfoncement par coup

$f_{0} \quad$ : fiche initiale

f. : fiche finale

$\Delta t \quad$ : intervalle entre 2 coups

Q : capacité portante

k : nombre d'allers-retours de l'onde

Indices

$\mathrm{m}:$ marteau
$\mathrm{p} \quad$ : pieu
$0 \quad$ : choc théorique
$\max :$ valeur maximale mesurée

Exposant

* : facteur d'échelle

\section{Introduction}

L'intérêt de l'utilisation de la centrifugation pour réaliser des essais dans les divers domaines de la géotechnique n'est plus à démontrer; coûts réduits par rapport à une expérimentation sur site, possibilité de maitriser au mieux les caractéristiques du sol et d'effectuer des études paramétriques, résultats plus représentatifs que ceux obtenus sur des modèles réduits non centrifugés..

Parmi les domaines d'étude [1, 2] figurent les fondations profondes isolées ou en groupe, soumises à divers types de sollicitation : chargement axial, chargement latéral, chargement monotone ou cyclique, voire dynamique. Les pieux moulés ne représentent qu'un faible pourcentage des pieux installés sur site, tout au moins en France. Par ailleurs, le mode de mise en place par battage est quasiment la seule technique d'installation pour les travaux offshore. Or jusqu'ici, l'essentiel des essais centrifugés pour l'étude du comportement des pieux portent sur des pieux moulés ou sur des pieux préalablement battus à $1 \mathrm{~g}$.

L'utilisation de pieux moulés centrifugés s'explique en grande partie par une mise en œuvre relativement aisée pour l'expérimentateur qui utilise la pluviation comme méthode de remplissage dans le cas de matériaux granulaires. Ces pieux-modèles ne sont pas représentatifs des pieux battus, ne serait-ce que par la densification du sol autour du pieu due au refoulement du sol par le pieu lors de sa pénétration.

Tester des pieux-modèles préalablement battus à $1 \mathrm{~g}$ n'est guère plus satisfaisant, car lors de leur installation, ces pieux-modèles sont soumis à un champ de contraintes très faibles, et donc très différent de celui appliqué aux pieux-prototypes.

Il est donc tout à fait évident que l'étude relative au comportement des pieux battus impose que les pieuxmodèles le soient aussi en cours de centrifugation. Ce mode de mise en œuvre nécessite non seulement de disposer d'un batteur embarquable, mais aussi que l'on ait vérifié expérimentalement la validité des lois de similitude pour une exploitation fiable en vraie grandeur.

\section{2.}

\section{Relations de base de la similitude}

\section{Rappel des relations de base}

Les relations de base de la similitude proviennent directement de l'équation générale de la mécanique:

$$
\sum_{i} \frac{\partial \sigma_{i j}}{\partial x_{j}}+\rho\left(g_{i}+\frac{\partial^{2} u_{i}}{\partial t^{2}}\right)=0
$$

avec:

$\sigma:$ tenseur des contraintes,

$x$ : coordonnée,

u: déplacement,

$p$ : masse volumique,

$\mathrm{g}$ : accélération de la pesanteur,

t: temps. 
Pour le prototype, les variables seront notées sans désignation particulière, par exemple $\sigma$ pour les contraintes. Pour le modèle, les variables seront notées avec un " *, par exemple $\sigma$ '. Les facteurs d'échelle, c'est-à-dire le rapport de la valeur modèle à la valeur prototype, seront désignés par un astérisque, par exemple:

$$
\sigma^{*}=\sigma^{\prime} / \sigma
$$

De l'équation (1) découle la double condition:

$$
\mid \begin{aligned}
& \sigma^{*}=\rho^{*} g^{*} x^{*} \\
& \mathrm{u}^{*}=\mathrm{g}^{*} \mathrm{t}^{* 2}
\end{aligned}
$$

Dans les essais centrifugés, on utilise en général les mêmes matériaux pour le modèle et pour le prototype, et on s'efforce de conserver la masse volumique:

$$
\rho^{*}=1
$$

Par ailleurs, on se limitera à la similitude simple :

$$
u^{*}=\mathrm{x}^{*}
$$

et on désignera par:

$$
\mathrm{g}^{*}=\mathrm{N}
$$

le facteur d'échelle de la gravité.

En ce qui nous concerne ici, le batteur est du type marteau à chute libre. Les caractèristiques du battage sont dans ce cas directement reliées à l'énergie $E_{0}$ du marteau et à sa vitesse $\mathrm{v}_{0}$ de chute juste avant le contact avec le pieu.

$$
E_{0}=M_{m} g H=\frac{1}{2} M_{m} v_{0}^{2}
$$

$\mathrm{M}_{\mathrm{m}}$ désigne la masse du marteau et $\mathrm{H}$ sa hauteur de chute.

On sait par ailleurs que lors du passage d'une onde de contrainte dans un milieu monodimensionnel élastique linéaire, la contrainte $\sigma$ est une fonction linéaire de la vitesse de déplacement particulaire $\mathrm{v}$ :

$$
\sigma=\mathrm{z} v+\mathrm{b}
$$

où z désigne l'impédance du milieu monodimensionnel et où b est une constante dépendant de l'état de contrainte avant le passage de la perturbation. L'impédance du milieu et la vitesse de propagation des ondes sont données par:

$$
\mid \begin{aligned}
& z=\sqrt{\overline{\bar{E}} \rho} \\
& c=\sqrt{\bar{E}} / \rho
\end{aligned}
$$

E désignant le module d'élasticité du milieu. On en déduit les facteurs d'échelle $\mathrm{z}^{*}$ et $\mathrm{c}^{*}$ lorsque le pieumodèle et le pieu-prototype sont constitués du même matériau.

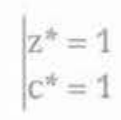

\section{2,2}

\section{Exploitation des relations de base}

Ces relations seront utilisées dans deux cas différents:
- Dans le premier cas, le pieu-modèle, le marteaumodèle et sa hauteur de chute sont identiques pour l'ensemble des essais; seul change le facteur d'échelle de la gravité. La relation (7) permet de déduire que dans ces conditions, les facteurs d'échelle $E^{*}$ des énergies et les facteurs d'échelle $\mathrm{v}^{*}$ des vitesses sont définies par:

$$
\mid \begin{aligned}
& E^{*}=N \\
& v^{*}=N^{1 / 2}
\end{aligned}
$$

Les autres relations montrent que l'on ne peut pas respecter tous les facteurs d'échelle. Entre autres, on n'aura pas le même facteur d'échelle pour la vitesse de déplacement particulaire $\left(\mathrm{v}^{*}=\mathrm{N}^{1 / 2}\right)$ et pour la vitesse de propagation des ondes dans le pieu $\left(c^{*}=1\right)$. L'analyse ne pourra donc porter que sur certains éléments particuliers, dont la force et l'accélération en tête de pieu pendant l'impact.

On trouvera dans le tableau I les principaux facteurs d'échelle exploitables en tête de pieu.

TABLEAUI Principaux facteurs d'échelle (cas 1). Main scale factors (case 1).

\begin{tabular}{c|c|c}
\hline Grandeur & Notation & Facteur \\
\hline énergie & $\mathrm{E}^{*}$ & $\mathrm{~N}$ \\
\hline vitesse particulaire & $\mathrm{v}^{*}$ & $\mathrm{~N}^{1 / 2}$ \\
\hline force en tête & $\mathrm{F}^{*}$ & $\mathrm{~N}^{1 / 2}$ \\
\hline gravité ou accélération & $\mathrm{g}^{*}$ ou a* & $\mathrm{N}$ \\
\hline
\end{tabular}

- Dans le deuxième cas, on s'impose d'avoir le même niveau de contrainte sur le modèle et sur le prototype:

$$
\sigma^{*}=1
$$

Les relations (3) à (10) permettent de définir les autres facteurs d'échelle sans rencontrer les difficultés du cas précédent.

On trouvera dans le tableau II les principaux facteurs d'échelle.

TABLEAU H Principaux facteurs d'échelle (cas 2). Main scale factors (case 2).

\begin{tabular}{c|c|c} 
Grandeur & Notation & Facteur \\
\hline contrainte & $\sigma^{*}$ & 1 \\
\hline masse volumique & $\rho^{*}$ & 1 \\
\hline gravité ou accélération & $\mathrm{g}^{*}$ ou a* & $\mathrm{N}$ \\
\hline longueur ou déplacement & $\mathrm{x}^{*}=\mathrm{u}^{*}$ & $\mathrm{~N}^{-1}$ \\
\hline temps & $\mathrm{t}^{*}$ & $\mathrm{~N}^{-1}$ \\
\hline énergie & $\mathrm{E}^{*}$ & $\mathrm{~N}^{-3}$ \\
\hline vitesse & $\mathrm{v}^{*}$ & 1 \\
\hline force & $\mathrm{F}^{*}$ & $\mathrm{~N}^{-2}$ \\
\hline masse & $\mathrm{M}^{*}$ & $\mathrm{~N}^{-3}$ \\
\hline
\end{tabular}

\section{3}

\section{Première vérification expérimentale}

La première série d'essais a été réalisée à l'aide de la centrifugeuse de I'Université de Bochum, dans le cadre du programme de coopération franco-allemand PRO- 
COPE associant cette Université à l'École Centrale de Nantes $[3,4]$. On ne retiendra ici que les résultats nécessaires à la vérification des lois de similitude exposées plus haut.

\section{1}

\section{Dispositif expérimental}

La figure 1 présente le schéma du dispositif expérimental. Le dispositif de battage est réduit à sa plus simple expression: il s'agit d'un système pendulaire n'autorisant qu'un seul choc en vol, la centrifugeuse devant être arrêtée après chaque choc pour réarmer le dispositif. Le pieu en duralumin est du type moulé. Il est presque entièrement fiché dans du sable fin sec. Un certain nombre de capteurs a été mis en place : nous ne nous intéresserons ici qu'à la station de jauges de déformation et à l'accéléromètre installés au sommet du pieu.

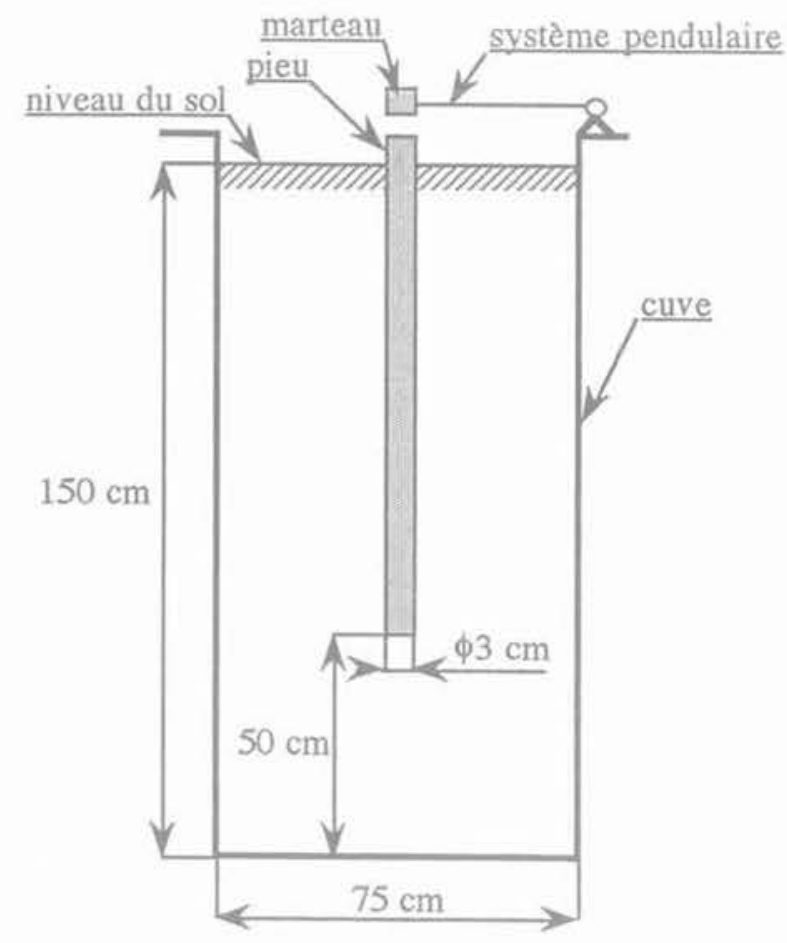

FIG.1 Dispositif expérimental (Bochum). Experimental set-up (Bochum).

Le sable sec dont la taille des grains va de 0,06 à $1,2 \mathrm{~mm}$, est mis en place par pluviation.

L'ensemble des valeurs caractéristiques sont rassemblées dans le tableau III.

Plusieurs impacts ont été réalisés à $1 \mathrm{~g}, 20 \mathrm{~g}$ et $30 \mathrm{~g}$. Il leur correspond des vitesses respectives de chute du marteau juste avant contact de $0,63,2,80$ et $3,43 \mathrm{~ms}^{-1}$.

\section{2}

\section{Force en tête}

On trouvera sur la figure 2 l'évolution de la force en tête pendant le choc en fonction du temps pour chacun des niveaux de gravité.
TABLEAU III Principales caractéristiques expérimentales.

Main experimental set-up characteristics.

\begin{tabular}{c|c}
\hline diamètre interne de la cuve & $75 \mathrm{~cm}$ \\
\hline hauteur de remplissage & $150 \mathrm{~cm}$ \\
\hline poìds volumique du sol & $15,21 \mathrm{kN} / \mathrm{m}^{3}$ \\
\hline densité relative & $65 \%$ \\
\hline longueur du pieu & $105 \mathrm{~cm}$ \\
\hline diamètre du pieu & $30 \mathrm{~mm}$ \\
\hline fiche du pieu & $100 \mathrm{~cm}$ \\
\hline masse du marteau & $1300 \mathrm{~g}$ \\
\hline hauteur de chute du marteau & $20 \mathrm{~mm}$ \\
\hline
\end{tabular}

On observe:

- d'une part que la force d'impact augmente avec le niveau de gravité;

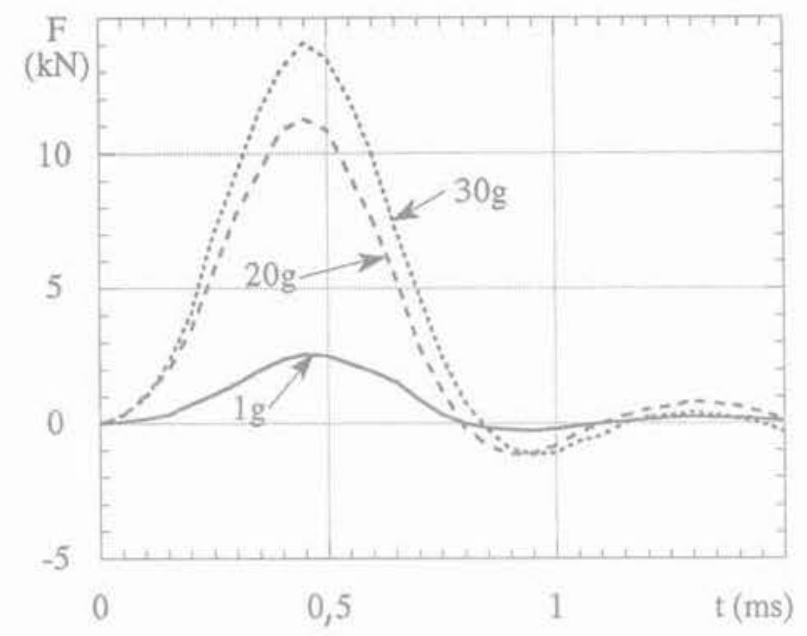

FG. 2. Forces d'impact. Impact forces.

- d'autre part que la durée du contact entre le marteau et le pieu ainsi que l'instant où la force maximale est obtenue ne varient pas de façon significative avec le niveau de gravité. De façon plus précise, on peut calculer le facteur d'échelle $\mathrm{F}^{*}$ de la force d'impact suivant le tableau I pour la valeur maximale de la force d'impact. On trouvera les résultats dans le tableau IV.

Les valeurs $\mathrm{F}_{\max }$ correspondent aux valeurs maximales lues sur la figure 2. Les facteurs d'échelle $\mathrm{F}^{*}$ ont été calculés par la relation du tableau I en prenant pour référence la valeur obtenue pour $\mathrm{N}=1$. Les facteurs d'échelle $\mathrm{F}^{*}$ ont été calculés en faisant directement le rapport entre $\mathrm{F}_{\max }$ à Ng et $\mathrm{F}_{\max }$ à $1 \mathrm{~g}$. On constate l'excellent accord entre les facteurs d'échelle théoriques et expérimentaux, l'écart étant inférieur à 1,6\%. 
TABLEAUIN Facteurs d'échelle de la force d'impact. Impact force scale factors.

\begin{tabular}{c|c|c|c}
$N$ & $F_{\max }(k N)$ & $F_{j}^{*}$ & $F_{\text {exp }}^{*}$ \\
\hline 1 & 2,56 & 1 & 1 \\
\hline 20 & 11,26 & 4,47 & 4,40 \\
\hline 30 & 14,08 & 5,48 & 5,50
\end{tabular}

A partir de ces résultats, on peut ramener les trois courbes $F(t)$ au même niveau de référence à $1 \mathrm{~g}$. Il suffit pour cela de calculer:

$$
F^{\prime}(t)=F(t) / F^{*}
$$

On trouvera sur la figure 3 les résultats ainsi obtenus.

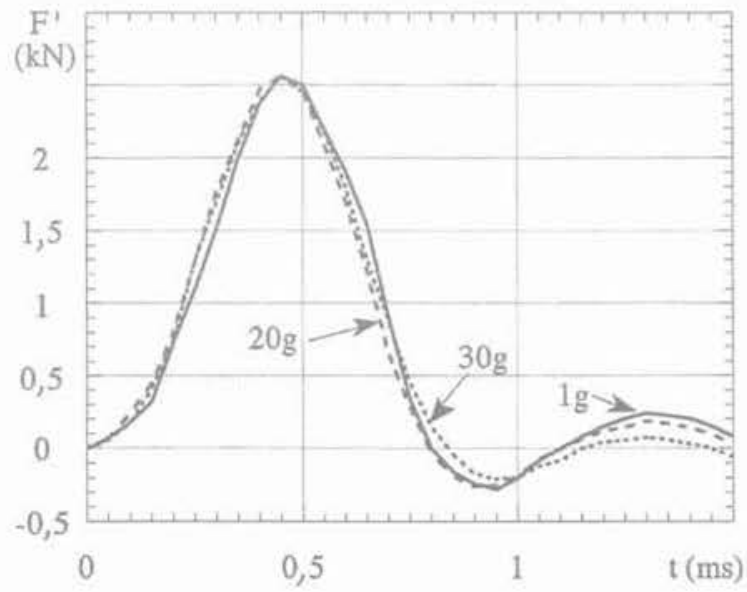

FG.3 Forces d'impact réduites à $1 \mathrm{~g}$. Impact forces scaled to $1 \mathrm{~g}$.

Les trois courbes sont extrêmement voisines les unes des autres, ce qui confirme que le facteur d'échelle des efforts est bien vérifié.

3.3

\section{Accélération en tête}

Les accélérations en tête ont été mesurées en même temps que les efforts d'impact. On observe très clairement sur les signaux obtenus lors des essais les divers allers-retours de l'onde. Pour les trois niveaux d'accélération, la durée séparant deux pics successifs est de $0,42 \mathrm{~ms}$, ce qui donne une vitesse de propagation de $5000 \mathrm{~ms}^{-1}$ correspondant bien aux caractéristiques du duraluminium.

On observe de plus que la décroissance du signal est d'autant plus rapide que le niveau $\mathrm{Ng}$ est grand. Cette décroissance étant représentative de l'énergie dissipée par frottement le long du fût du pieu, on vérifie bien qualitativement que les contraintes appliquées par le sol sur le pieu augmentent avec le niveau de gravité.

On peut calculer le facteur d'échelle a* suivant le tableau I pour la valeur maximale de l'accélération. On trouvera les résultats dans le tableau V.

Les valeurs $a_{\max }$ correspondent aux valeurs maximales des signaux d'accélération. Les facteurs d'échelle $\mathrm{a}^{\star}{ }_{0}$ ont été calculés par la relation du tableau I en prenant pour référence la valeur obtenue pour $N=1$. Les
TABLEAUV Facteurs d'échelle de l'accélération maximale en tête.

Pile top maximum acceleration scale factors.

\begin{tabular}{c|c|c|c}
\hline$N$ & $a_{\max }(g)$ & $a^{*}$ & $a^{*}=1$ \\
\hline 1 & 107 & 1 & 1 \\
\hline 20 & 2143 & 20 & 20,0 \\
\hline 30 & 3214 & 30 & 30,0 \\
\hline
\end{tabular}

facteurs d'échelle $a^{*}$ ont été calculés en faisant directement le rapport entre $\mathrm{a}_{\max }$ à $\mathrm{Ng}$ et $\mathrm{a}_{\max }$ à $1 \mathrm{~g}$. On constate l'excellent accord entre les facteurs d'échelle théoriques et expérimentaux, l'écart n'étant pas décelable.

En conclusion, bien que dans le cas présent toutes les lois de similitude ne soient pas respectées, on constate que les facteurs d'échelle de la force d'impact et ceux de l'accélération maximale en tête sont parfaitement vérifiés.

\section{Deuxième vérification expérimentale}

La deuxième série d'essais a été réalisée à l'aide de la centrifugeuse du Laboratoire Central des Ponts et Chaussées de Nantes dans le cadre du programme Macrogravité du ministère de la Recherche. L'étude a été menée en association entre l'École Centrale de Nantes et I'Université de Nantes, et avec le concours du LCPC $[5,6]$

\section{1}

\section{Objectifs de l'étude}

L'objectif de cette étude était précisément de vérifier expérimentalement la validité des lois de similitude. Pour cela, on a décidé de tester des pieux-modèles de dimensions différentes sous des niveaux de gravité tels qu'ils représentent tous le même prototype.

Le programme comprenait deux phases:

- le battage des pieux en cours de centrifugation, avec le relevé des courbes de battage et l'enregistrement des ondes induites dans le pieu par l'impact;

- la mesure de la capacité portante des pieux après battage.

Des tests de capacité portante ont été également réalisés sur un pieu moulé et sur un pieu battu à $1 \mathrm{~g}$ pour mettre en évidence la différence de comportement avec les pieux battus en cours de centrifugation.

\section{2}

\section{Facteurs d'échelle}

On trouvera dans les tableaux VI et VII l'ensemble des facteurs d'échelle à respecter pour le marteau, le pieu et le choc, les indices m et p désignant respectivement le marteau et le pieu.

De façon à simplifier au maximum les caractéristiques du choc, on a choisi dans chaque cas d'utiliser un marteau et un pieu de même diamètre et constitués 
TABtEAU VI Facteurs d'échelle communs au marteau et au pieu.

Pile and hammer scale factors.

\begin{tabular}{c|c|c}
\hline Grandeur & Notation & Facteur \\
\hline longeur & $\mathrm{L}_{\mathrm{m}}^{*}-\mathrm{L}_{\mathrm{p}}^{*}$ & $\mathrm{~N}^{-1}$ \\
\hline diamétre & $\phi_{\mathrm{m}}{ }^{*}-\phi_{\mathrm{p}}^{*}$ & $\mathrm{~N}^{-1}$ \\
\hline masse & $\mathrm{M}_{\mathrm{m}}^{*}-\mathrm{M}_{\mathrm{p}}^{*}$ & $\mathrm{~N}^{-3}$ \\
\hline vitesse de propagation des ondes & $\mathrm{c}_{\mathrm{m}}^{*}-\mathrm{c}_{\mathrm{p}}^{*}$ & 1 \\
\hline durée d'un aller-retour & $\tau_{\mathrm{m}}^{*}-\tau_{\mathrm{p}}^{*}$ & $\mathrm{~N}^{-1}$ \\
\hline impédance & $\mathrm{z}_{\mathrm{m}}^{*}-\mathrm{z}_{\mathrm{p}}^{*}$ & 1 \\
\hline
\end{tabular}

TABLEAU BII Facteurs d'échelle du choc et du battage. Driving and impact scale factors:

\begin{tabular}{c|c|c} 
Grandeur & Notation & Facteur \\
\hline hauteur de chute du marteau & $\mathrm{H}^{*}$ & $\mathrm{~N}^{-1}$ \\
\hline vitesse de chute avant impact & $\mathrm{V}_{0}^{*}$ & 1 \\
\hline durée du choc & $\tau^{*}$ & $\mathrm{~N}^{-1}$ \\
\hline contrainte induite & $\sigma^{*}$ & 1 \\
\hline force induite & $\mathrm{F}^{*}$ & $\mathrm{~N}^{-2}$ \\
\hline vitesse particulaire & $\mathrm{v}^{*}$ & 1 \\
\hline énergie & $\mathrm{E}^{*}$ & $\mathrm{~N}^{-3}$ \\
\hline enfoncement par coup & $\delta^{*}$ & $\mathrm{~N}^{-1}$ \\
\hline nombre de coups & $\mathrm{n}^{*}$ & 1 \\
\hline cadence de battage & $\mathrm{f}^{*}$ & $\mathrm{~N}$ \\
\hline
\end{tabular}

du même matériau de module d'élasticité Eet de masse volumique $\rho$. On a donc les relations suivantes:

$$
\begin{aligned}
& \phi_{m}=\phi_{p}=\phi \\
& c_{m}=c_{p}=c=\sqrt{\bar{E} / p} \\
& z_{m}=z_{p}=z=\sqrt{\bar{E} p}
\end{aligned}
$$

On peut par ailleurs expliciter les durées des allersretours des ondes dans le marteau et dans le pieu:

$$
\mid \begin{aligned}
& \tau_{m}=2 L_{m} / c \\
& \tau_{p}=2 L_{p} / c
\end{aligned}
$$

\section{3}

\section{Dispositif expérimental}

La figure 4 présente le schéma du dispositif expérimental.

\subsection{5}

\section{Principe de fonctionnement du batteur}

Le dispositif comporte un servo-vérin dont le corps est fixe. Le corps du batteur (ou guide) est lié rigidement à la tige du vérin. Un capteur de force est installé entre ces deux éléments. A l'intérieur du corps du batteur qui sert également de guide à la fois pour le marteau et pour le pieu - est fixée une ventouse électromagnétique.

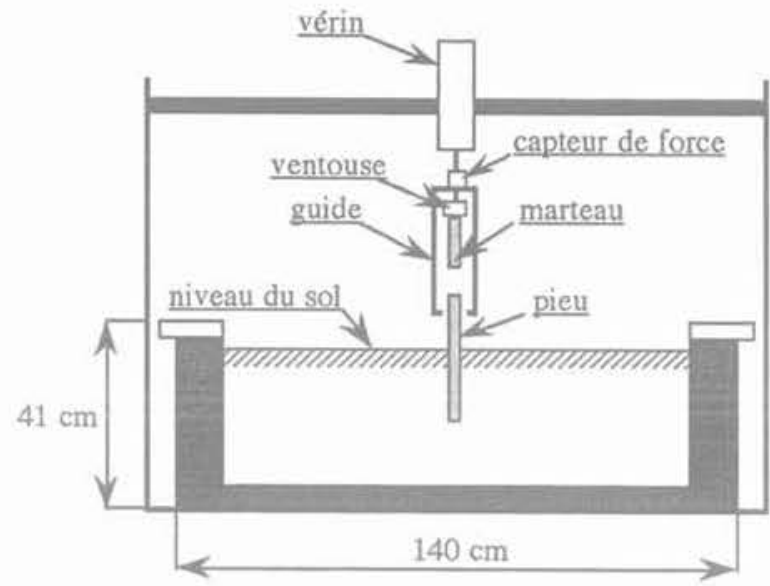

FG. 4 Dispositif expérimental (Nantes). Experimental set-up (Nantes).

Lorsque le marteau est en contact avec le pieu, le vérin descend l'ensemble corps du batteur et ventouse. Un système de diodes opto-électroniques solidaires du corps du batteur, permet de déceler le contact de la ventouse avec le marteau. La ventouse est alors activée et la tige du vérin remonte, entrainant la ventouse et le marteau.

Un deuxième système de diodes opto-électroniques solidaires du corps du batteur, permet de déceler la position haute du marteau. La ventouse est alors désactivée, et le marteau tombe sous son propre poids. On entame alors le cycle suivant. Un capteur de déplacement solidaire de la tige du vérin permet d'enregistrer le déplacement du corps du batteur, et donc de connaitre les positions basses et hautes du marteau. La fiche du pieu est déterminée à partir de la position basse du marteau par un changement d'origine approprié.

Pour mesurer la capactié portante du pieu en statique, il suffit de descendre la tige du vérin, qui dans son mouvement appliquera un effort statique sur le pieu par l'intermédiaire du marteau, de la ventouse et du capteur de force. Il est donc aisé de relever la courbe force-enfoncement.

La partie inférieure du corps du batteur est interchangeable, de façon à être adaptée au diamètre du pieu et du marteau.

\section{Caractéristiques des pieux et des marteaux}

Pour définir les caractéristiques des pieux-modèles et des marteaux-modèles, on a préalablement choisi des caractéristiques géométriques réalistes du pieuprototype. Les caractéristiques du marteau-prototype ont été définies par des considérations énergétiques. Nous imposons que le pieu et le marteau aient même section et soient constitués du même matériau. On trouvera les valeurs de base dans le tableau VIII.

TABtEAU vil Caractéristiques de base du pieu et du marteau-prototype.

Prototype pile and hammer characteristics.

\begin{tabular}{c|c}
\hline longueur du pieu & $12,50 \mathrm{~m}$ \\
\hline diamètre du pieu & $0,50 \mathrm{~m}$ \\
\hline masse du marteau & $5000 \mathrm{~kg}$ \\
\hline hauteur de chute & $1,00 \mathrm{~m}$ \\
\hline énergie théorique & $49,1 \mathrm{~kJ}$ \\
\hline
\end{tabular}


Compte tenu des impératifs liés à l'encombrement du système, à la course du vérin et à la hauteur de sable dans la cuve, le plus grand pieu-modèle ne peut avoir une Iongueur supérieure à $400 \mathrm{~mm}$. Les caractéristiques des pieux et des marteaux-modèles retenus sont données dans le tableau IX. au voisinage immédiat de la tête. Aussi les stations de jauges ont elles dû être positionnées à une distance ( $\left.I_{1}\right)$ relativement importante du sommet du pieu (voir Fig. 5). Cette implantation nécessite une analyse particulière indispensable pour interpréter correctement les signaux de contrainte enregistrés au cours des essais.

TABLENU IX Caractéristiques des pieux et des marteaux-modèles. Model pile and hammer characteristics.

\begin{tabular}{c|c|c|c|c}
\hline niveau de gravité & 31,2 & 41,7 & 50,0 & 62,5 \\
\hline diamètre du pieu et du marteau (mm) & 16 & 12 & 10 & 8 \\
\hline longueur du pieu $(\mathrm{mm})$ & 400 & 300 & 250 & 200 \\
\hline longueur du marteau $(\mathrm{mm})$ & 104,5 & 78,3 & 65,3 & 52,2 \\
\hline hauteur de chute du marteau $(\mathrm{mm})$ & 32 & 24 & 20 & 16 \\
\hline masse du marteau $(\mathrm{g})$ & 163,8 & 69,1 & 40,0 & 20,5 \\
\hline énergie théorique $(\mathrm{J})$ & 1,607 & 0,678 & 0,392 & 0,211 \\
\hline
\end{tabular}

Les pieux et les marteaux sont en acier Stub de masse volumique $7800 \mathrm{~kg} / \mathrm{m}^{3}$.

\subsubsection{5}

\section{Caractéristiques du sol}

Le sol est un sable blanc de Fontainebleau, de granulométrie serrée $(0,08$ à $0,8 \mathrm{~mm})$. Il est mis en place par pluviation. Ses principales caractéristiques sont rassemblées dans le tableau X. Il s'agit en fait d'un sable propre mal gradué.

TABLEAUX Caractéristiques du sol. Soil characteristics.

\begin{tabular}{c|c}
\hline coefficient de Hazen & 1,54 \\
\hline coefficient de courbure & 0,98 \\
\hline poids volumique & $14,75 \mathrm{kN} / \mathrm{m}^{3}$ \\
\hline densité relative & $36 \%$ \\
\hline
\end{tabular}

Le diamètre moyen des grains est de $0,19 \mathrm{~mm}$. On respecte donc la condition généralement admise que le diamètre du pieu soit supérieur à 30 fois le diamètre moyen des grains pour ne pas avoir d'effet d'échelle entre la taille des grains et le diamètre du pieu-modèle,

\section{4}

\section{Analyse des ondes de contrainte}

\section{Cactili \\ Calculs préliminaires}

Tous les pieux testés étaient équipés d'une station de jauges de déformation. L'idéal eût été de placer ces stations de jauges le plus près possible de la tête du pieu. Le dispositif de battage servant également de guidage du pieu, il n'était pas possible de mettre les jauges
Dans toute la suite, on utilisera les notations suivantes:

$-I_{1}$ : distance de la station de mesure à la tête du pieu; $-\mathrm{I}_{2}$ : distance de la station de mesure à la base du pieu; $-\mathrm{L}_{e}$ : longueur de la perturbation induite par le choc; - c: vitesse de propagation des ondes dans le pieu et dans le marteau.

Dans le cas du choc parfait entre un pieu et un marteau tombant en chute libre, de même matériau et de même section, l'ébranlement induit en tête de pieu est caractérisé par:

- une durée $\tau_{0}$ égale à celle d'un aller-retour de l'onde dans le marteau (relation 15), ce qui se traduit par:

$$
\begin{aligned}
& \tau_{0}=\tau_{m} \\
& L_{e}=2 L_{m}
\end{aligned}
$$

- une contrainte constante $\sigma_{0}$ pendant toute la durée du choc directement proportionnelle à la vitesse de chute $v_{0}$ du marteau avant l'impact et à l'impédance $z$ du marteau et du pieu:

$$
\sigma_{0}=\frac{\mathrm{zV}_{0}}{2}
$$

Un choc réel n'est jamais parfait. Ceci se traduit à la fois par une durée de contact supérieure à $\tau_{0}$ et une contrainte variable pendant le choc.

On pourra se reporter à la figure 5 pour une meilleure compréhension de l'analyse qui va suivre. L'origine du temps a été choisie comme l'instant d'arrivée du premier front de l'onde au niveau de la station de mesure. Pour simplifier la représentation graphique des différentes étapes, on a représenté une perturbation de contrainte constante, mais le raisonnement peut être extrapolé à une perturbation de forme quelconque. Pour cette analyse, on a également supposé que le pieu est parfaitement libre (absence de sol). 


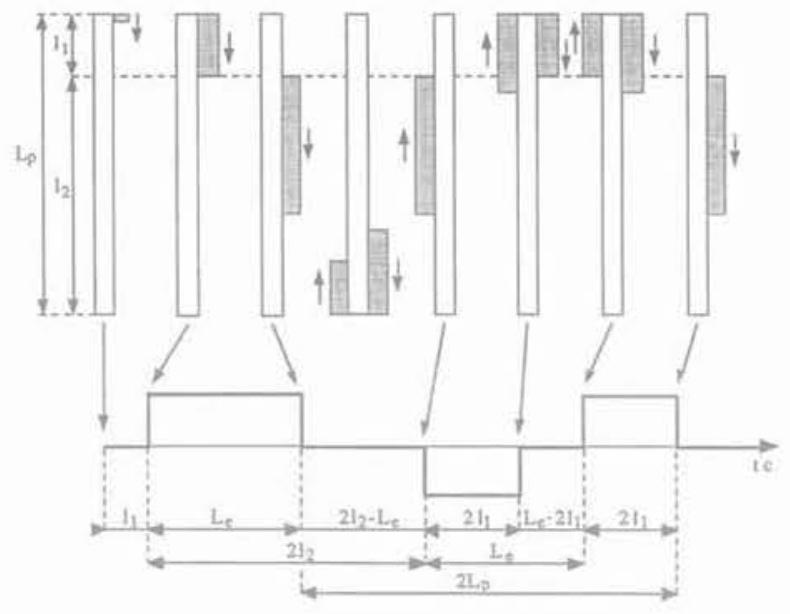

ตG.5 Analyse de la propagation des ondes. Stress-wave propagation analysis.
- Entre les instants $\mathrm{t}=0$ et $\mathrm{t}=\mathrm{L} / \mathrm{c}$, les jauges mesurent l'ébranlement initial (onde de compression) induit par le choc du marteau sur le pieu.

- Entre les instants $t=I_{2} / c$ et $t=\left(l_{2}+L_{2}\right) / c$, l'onde descendante de compression se réfléchit à la base du pieu pour donner naissance à une onde montante de traction.

- Le front de cette onde montante apparait au niveau de la station de mesure à l'instant $t=21 / \mathrm{c}$. Entre les instants $t=2 l_{2} / \mathrm{c}$ et $t=2 l_{p} / c$, les jauges mesurent uniquement cette onde montante.

- Entre les instants $t=\left(\mathrm{L}_{\mathrm{p}}+\mathrm{L}_{2}\right) / \mathrm{c}$ et $\mathrm{t}=\left(\mathrm{L}_{\mathrm{p}}+\mathrm{I}_{2}+\mathrm{L}_{\mathrm{e}}\right) / \mathrm{c}$, l'onde montante de traction se réfléchit au sommet du pieu pour donner naissance à une nouvelle onde descendante de compression.

- A l'instant $=2 \mathrm{~L}_{\mathrm{p}} / \mathrm{c}$, le front de cette nouvelle onde descendante apparait au niveau de la station de mesure. Entre les temps $t=2 L_{0} / c$ et $t=\left(2 l_{1}+L_{1}\right) / c$, on a donc dans cette section la superposition dé londe montante et de l'onde descendante.

- L'instant $\mathrm{t}=\left(2 \mathrm{I}_{2}+\mathrm{L}\right) / \mathrm{c}$ caractérise la fin du passage de l'onde montante au niveau de la station de mesure. Entre les instants $t=\left(2 L_{2}+L_{e}\right) / c$ et $t=\left(2 L_{n}+\right.$ $\mathrm{L}_{\mathrm{p}} / \mathrm{c}$, les jauges mesurent la seule onde descendante.

- A partir de $t=\left(2 L_{f}+L_{-}\right) / c$, le phénomène observé à partir de $\mathrm{l}^{\prime}$ instant $\mathrm{t}=\mathrm{L}_{e} / \mathrm{c}$ se reproduit identique à luimême.

Ce raisonnement suppose à la fois que la longueur de l'ébranlement est supérieure à 2l ${ }_{1}$ et inférieure à $2 \mathrm{l}_{2}$.

On constate donc que la durée séparant l'arrivée de la première et de la deuxième onde de compression ne correspond pas à la durée d'un aller-retour de l'onde dans le pieu. Par contre, cette durée de référence se retrouve entre la fin de la première et de la deuxième onde de compression comme entre l'arrivée de la première et de la deuxième onde de traction, et l'arrivée de la deuxième et de la troisième onde de compression. La mesure de la vitesse de propagation des ondes dans le pieu ne devra donc surtout pas être faite en utilisant les débuts (voire les maxima) des deux premières ondes de compression.
Les propos précédents illustrés sur la figure 5 peuvent être généralisés à une perturbation initiale de forme quelconque $\sigma_{1}(t)$ par les relations suivantes :

$$
\begin{aligned}
& t \in\left[\frac{2 l_{2}}{c}, \frac{2 L_{p}}{c}\right] \Rightarrow \sigma(t)=-\sigma_{1}\left(t-\frac{2 l_{2}}{c}\right) \\
& t \in\left[\frac{2 L_{p}}{c}, \frac{2 l_{2}+L_{c}}{c}\right] \Rightarrow \sigma(t)=-\sigma_{1}\left(t-\frac{2 l_{2}}{c}\right)+\sigma_{1}\left(t-\frac{2 L_{p}}{c}\right) \\
& t \in\left[\frac{2 l_{2}+L_{e}}{c}, \frac{2 L_{p}+L_{e}}{c}\right] \Rightarrow \sigma(t)=\sigma_{1}\left(t-\frac{2 L_{p}}{c}\right)
\end{aligned}
$$

La figure 6 présente un enregistrement-type. On retrouve très explicitement les résultats prévus. II apparait très clairement que la durée correspond au temps nécessaire à un aller-retour du pieu entre:

- la fin de la première et de la deuxième onde de compression:

- la fin de la deuxième et de la troisième onde de compression;

- le début de la première et de la deuxième onde de traction:

- le début de la deuxième et de la troisième onde de compression.

On vérifie également que l'intervalle entre le début de la première et de la deuxième onde de compression est nettement supérieur à $2 \mathrm{~L}_{\mathrm{p}} / \mathrm{c}$.

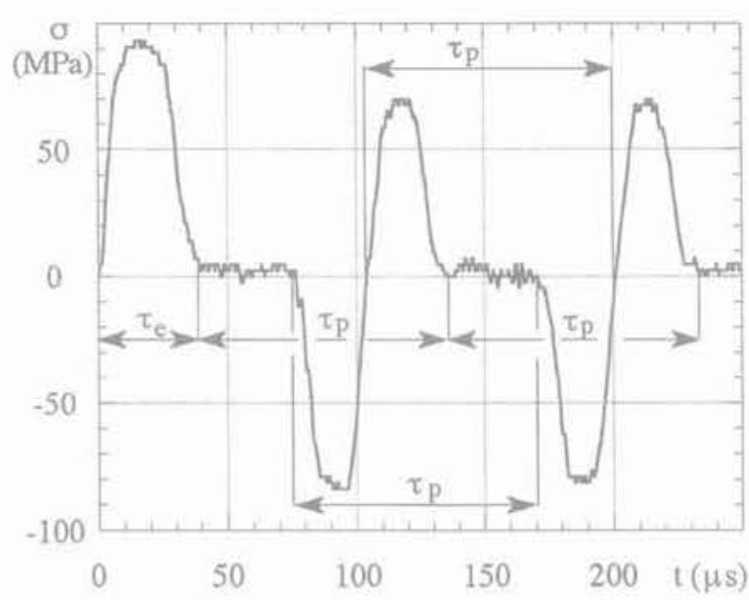

Fด. 6 Enregistrement-type.

Typical signal record.

A partir de ce qui précède, en analysant l'ensemble des signaux enregistrés pour tous les pieux testés, on obtient une vitesse moyenne de propagation des ondes dans les pieux de $5170 \mathrm{~m} / \mathrm{s}$.

Connaissant la masse volumique de l'acier ainsi que les conditions de la chute du mouton, on peut alors calculer l'ensemble des grandeurs caractéristiques communes à tous les pieux par application des relations (14) et (17). Les valeurs correspondantes ont été regroupées dans le tableau XI ci-dessous. 
4649

\section{Analyse des signaux}

Les jauges de déformation ont été placées sur l'ensemble des pieux de sorte que $l_{2} / L_{p}$ ait toujours la même valeur de 0,2 . On trouvera sur la figure 7 un exemple représentatif des mesures obtenues en fin d'essai pour chaque type de pieu. En cours de battage, ce sont en fait 5 à 6 mesures qui ont été réalisées pour chaque pieu. Il n'y a pas de différences significatives des signaux en fonction de la fiche. De façon à rendre la comparaison en fonction du temps plus aisée, les échelles des temps ont été choisies proportionnelles à la dimension des pieux.

L'oscilloscope à mémoire, dont nous nous sommes servis, a une résolution de $32 \mathrm{mV}$, ce qui correspond à environ $2,3 \mathrm{MPa}$ en unités physiques. Les irrégularités que l'on note sur l'ensemble des courbes correspond à cette résolution.

Compte tenu de ce qui a été dit au \$ 4.4.1, on peut définir la valeur maximale mesurée $\sigma_{\text {max }}$ de la contrainte pendant le choc, et la comparer avec la contrainte $\sigma$ du choc parfait. Les résultats pour les quatre pieux sont rassemblés dans le tableau XII ci-dessous. Les essais sont repérés par le diamètre du pieu (16, 12, 10 et $8 \mathrm{~mm}$ ) et par "a» pour le premier essai et $(\mathrm{b}$ ) pour le second essai pour un diamètre donné.

Compte tenu de la précision des mesures, les contraintes maxima sont tout à fait comparables à la contrainte du choc parfait.

Dans ce même tableau, on a reporté des durées $\tau$. du choc parfait ainsi que les durées $\theta$ des paliers pendant lesquels la contrainte maximale $\sigma_{\text {max }}$ reste constante. Si l'on note bien que ces durées diminuent avec la dimension des pieux, elles ne sont pas proportionnelles à $\tau_{\text {. }}$. Ce fait est à rapprocher d'un temps de montée qui est du même ordre de grandeur pour tous les pieux, ce qui réduit d'autant la longueur du palier.

On trouvera également dans le tableau XII la durée $\tau$ du choc réel. On notera une durée du choc anormalement longue pour le pieu de $8 \mathrm{~mm}$ de diamètre. Un autre essai réalisé également sur un pieu de diamètre $8 \mathrm{~mm}$ a donné une durée de $41,0 \mu \mathrm{s}$, mais un niveau de contrainte nettement supérieur à la contrainte théorique $(117 \%)$. Il apparaît clairement que le pieu de $8 \mathrm{~mm}$ de diamètre se dìstingue des trois autres pieux, à la fois par la durée relative du palier et par la durée relative du choc proprement dit.

TABLEAUXI Valeurs caractéristiques. Specific values.

\begin{tabular}{c|c|c|c|c|c}
\hline$c\left(\mathrm{~ms}^{-1}\right)$ & $\rho\left(\mathrm{kg} \cdot \mathrm{m}^{-3}\right)$ & $\overline{\mathrm{E}}(\mathrm{MPa})$ & $2\left(\mathrm{~Pa} \mathrm{~m} \mathrm{~m}^{-1} \mathrm{~s}\right)$ & $\nabla_{0}\left(\mathrm{~ms}^{-1}\right)$ & $\sigma_{0}(\mathrm{MPa})$ \\
\hline 5170 & 7800 & $2,085.10^{5}$ & $40,33.10^{6}$ & 4,43 & 89,3 \\
\hline
\end{tabular}
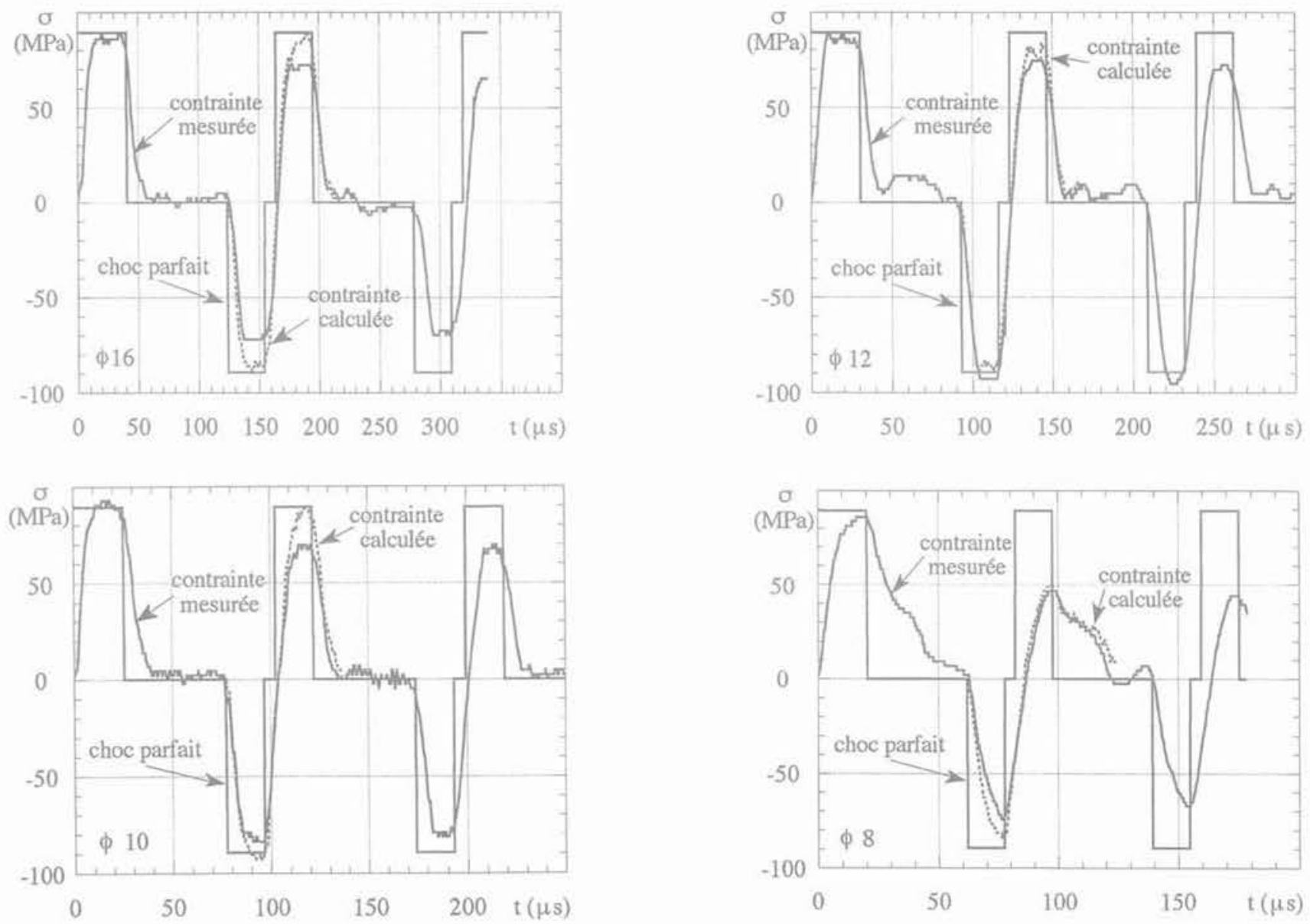

F6. 7 Ondes de contrainte.

Stress-waves. 
TABLEAUXII Caractérisations des chocs.

Impact characteristics.

\begin{tabular}{c|c|c|c|c|c|c|c}
\hline pieu & $\sigma_{0}$ (MPa) & $\sigma_{\operatorname{man}}(\mathrm{MPa})$ & $\tau_{0}(\mu \mathrm{s})$ & $\theta(\mu \mathrm{s})$ & $\tau(\mu \mathrm{s})$ & $\theta / \tau_{0}$ & $\tau / \tau_{0}$ \\
\hline $16 \mathrm{a}$ & 89,3 & $86,0 \pm 2,3$ & 40,4 & 22,4 & 56,4 & 0,554 & 1,40 \\
\hline $12 \mathrm{a}$ & 89,3 & $86,0 \pm 2,3$ & 30,3 & 17,7 & 44,1 & 0,584 & 1,46 \\
\hline $10 \mathrm{a}$ & 89,3 & $90,6 \pm 2,3$ & 25,3 & 12,3 & 39,6 & 0,486 & 1,57 \\
\hline $8 \mathrm{~b}$ & 89,3 & $86,0 \pm 2,3$ & 20,2 & 4,6 & 60,6 & 0,228 & 3,00 \\
\hline
\end{tabular}

On a également reporté sur ces mêmes figures 7 l'onde de contrainte théorique clans le cas du choc parfait. Dans tous les cas, il y a très bonne concordance entre les instants de début des ondes de traction. Cette concordance est également très bonne pour les pieux. de diamètre 16,12 et $10 \mathrm{~mm}$ en ce qui concerne le début des ondes de compression.

On trouvera sur ces mêmes figures 7 la représentation de la première onde de traction et de la deuxième onde de compression calculées par les relations (18) à partir de la première onde de compression mesurée. On constate là encore une excellente concordance sur la forme, et ceci pour les quatre pieux testés, y compris le pieu de diamètre $8 \mathrm{~mm}$. Les perturbations que l'on relève essentiellement au niveau des pics proviennent des perturbations de l'onde initiale que l'on n'a volontairement pas lissée.

Ces ondes ayant été calculées sans tenir compte de la présence du sol, la différence d'amplitude entre les ondes calculées et les ondes mesurées représente l'influence du frottement latéral et de l'effort de pointe. La présence du sol a pour effet de diminuer les amplitudes des ondes de contrainte. On vérifie bien dans le cas des pieux de diamètre 16,10 et $8 \mathrm{~mm}$ que les amplitudes des ondes calculées sont supérieures aux amplitudes des ondes mesurées. C'est aussi le cas globalement pour la deuxième onde de compression pour le pieu de diamètre $12 \mathrm{~mm}$. Seule la première onde de traction pour ce même pieu ne vérifie pas cette propriété sans que nous ayons trouvé d'explication.

On notera aussi que les amplitudes maxima de l'onde calculée ne sont égales au maximum de l'onde initiale que si la durée du choc est suffisamment courte. C'est le cas pour les pieux de diamètre 16, 12 et $10 \mathrm{~mm}$, contrairement au pieu de diamètre $8 \mathrm{~mm}$ pour lequel la durée du choc est longue. Cela signifie qu'il ne suffit pas d'attribuer au sol seul la diminution des amplitudes de l'onde de contrainte, mais qu'il faut faire une analyse fine portant sur l'ensemble de l'onde initiale.

On trouvera dans le tableau XIII les diverses longueurs $2 \mathrm{I}_{1}, 2 \mathrm{I}_{2}$ et $\mathrm{L}_{t}$ qui permettent de vérifier que la condition:

$$
2 \mathrm{I}_{1} \leq \mathrm{L}_{\mathrm{e}} \leq 2 \mathrm{I}_{2}
$$

est bien vérifiée dans tous les cas, ce qui justifie l'utilisation des équations (18).

Il est intéressant de compléter la comparaison de la caractérisation des chocs par une analyse énergétique. L'énercie transmise peut être calculée à partir des ondes de contraintes:

$$
E=\int_{0}^{T} F v d t=\frac{S}{z} \int_{0}^{t} \sigma^{2} d t
$$

En l'absence d'onde montante pendant l'impact, la force en tête $\mathrm{F}$ et la vitesse particulaire en tête $\mathrm{v}$ sont directement proportionnelles. S désigne la section commune au pieu et au marteau.

On trouvera dans le tableau XIV ci-dessous:

- l'énergie transmise E calculée par la relation (20) pour chacun des pieux-modèles:

- l'énergie transmise $E_{p}$ calculée pour chacun des pieux-modèles mais ramenée au prototype;

- le rapport entre l'énergie transmise $E_{p}$ et l'énergie théorique $E_{0}$ du prototype dont la valeur a été donnée dans le tableau VII.

L'énergie transmise ramenée au prototype augmente lorsque le diamètre du pieu-modèle diminue comme le visualise la figure 8. Cette évolution ne peut pas s'expliquer uniquement par une éventuelle imprécision sur la hauteur de chute réelle du marteau.

TABLEAU XII Longueurs des chocs. Impact lengthes:

\begin{tabular}{c|c|c|c} 
pieu & $2 I_{1}(\mathrm{~mm})$ & $2 L_{2}(\mathrm{~mm})$ & $L_{-}(\mathrm{mm})$ \\
\hline 16 & 160 & 640 & 292 \\
\hline 12 & 120 & 480 & 228 \\
\hline 10 & 100 & 400 & 205 \\
\hline 8 & 80 & 320 & 313 \\
\hline
\end{tabular}

TABLEAUXiv Énergies. Energies.

\begin{tabular}{c|c|c|c} 
pieu & $E(J)$ & $E_{p}(k J)$ & $E_{p} / E_{0}(\%)$ \\
\hline $16 a$ & 1,283 & 39,2 & 79,8 \\
\hline $12 a$ & 0,565 & 40,9 & 83,3 \\
\hline $10 a$ & 0,361 & 45,1 & 92,0 \\
\hline $8 \mathrm{~b}$ & 0,196 & 47,8 & 97,6 \\
\hline
\end{tabular}

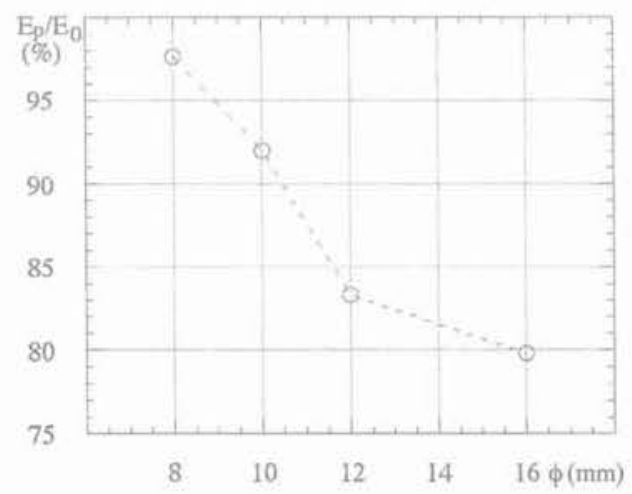

FIG. 8 Énergies transmises. Transmitted energies. 
Des enregistrements sur une durée relativement longue ( 30 allers-retours ou plus) ont été effectués pour les pieux de diamètre 16, 12 et $10 \mathrm{~mm}$. Dans les trois cas, les courbes sont très semblables. On trouvera à titre d'exemple sur la figure 9 l'enregistrement correspondant au pieu de diamètre $16 \mathrm{~mm}$.

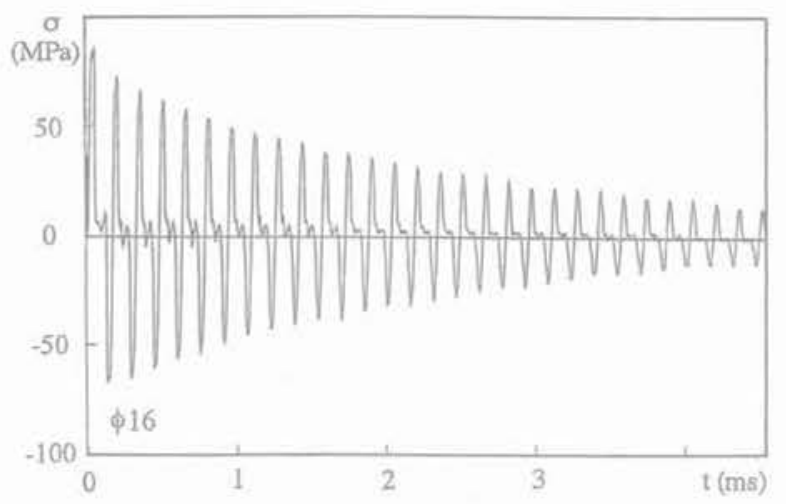

FiO. 9 Exemple d'amortissement. Typical damping signal record.

Rappelons que le premier pic positíf se distingue de tous les autres extrêma puisqu'il est le seul à ne pas être modifié par une éventuelle onde montante de traction (voir $\$ 4.4 .1$ ). Nous avons donc pris comme valeurs de référence l'amplitude du $2^{e}$ pic positif et du $1^{\text {er }}$ pic négatif qui prendront toutes deux le numéro 0. Pour les trois enregistrements, on a calculé les valeurs relatives des amplitudes des pics successifs en fonction du nombre d'allers-retours de l'onde. On a ensuite fait la moyenne pour chaque pieu de la ième valeur positive et de la ième valeur négative. Cette moyenne est justifiée par le fait que ces deux valeurs devraient être identiques dans la mesure oủ, entre ces deux pics, l'onde de contrainte ne se propage que dans la partie non fichée du pieu.

La figure 10 montre les résultats ainsi obtenus. Il apparait clairement que les résultats pour les trois pieux sont très voisins, ce qui indique que les comportements de ces trois pieux sont tout à fait comparables.

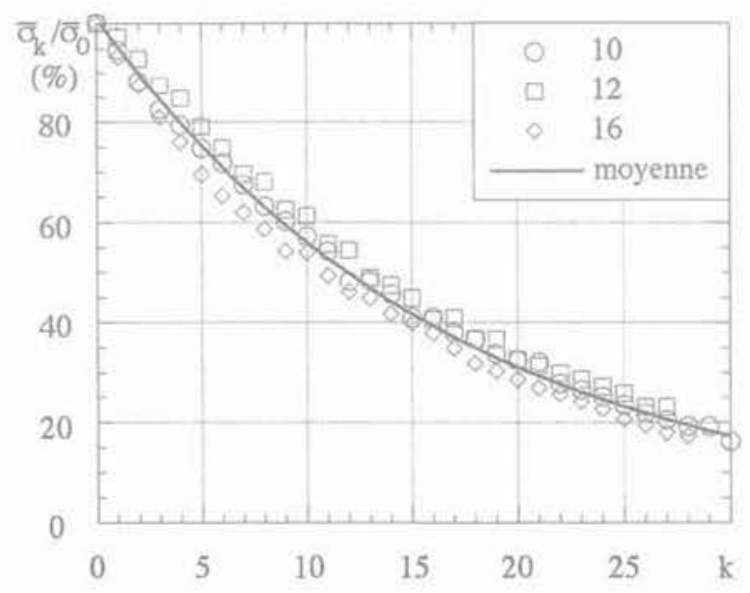

F16. 10 Caractérisation de l'amortissement. Characteristics of the damping.
Pour chacun des pieux, on a calculé la fonction exponentielle passant au mieux par l'ensemble des points sous la forme:

$$
\frac{\bar{\sigma}_{k}}{\bar{\sigma}_{0}}=\alpha \cdot \exp (-\beta k)
$$

où k désigne le nombre des allers-retours dans le pieu. Pour une meilleure lisibilité, on s'est limité à représenter sur la figure 10 la seule fonction exponentielle (désignèe par «moyenne » sur cette figure) correspondant à l'ensemble des trois pieux.

On trouvera dans le tableau XV ci-dessous les valeurs de $\alpha$ et de $\beta$ calculées par régression exponentielle, ainsi que la valeur du coefficient de corrélation r.

On constate que la corrélation pour chacun des pieux pris indépendamment est excellente. Elle est encore très bonne pour l'ensemble des trois pieux, ce qui confirme que les trois pieux ont un comportement très voisin.

On peut déduire un facteur d'amortissement partir de la valeur de $\beta$ par:

$$
\xi=\frac{\beta}{2 \pi}
$$

On obtient alors une valeur moyenne du facteur d'amortissement inférieure à $1 \%$. Cette valeur très petite est sans doute à relier à la faible compacité du sable.

De ce qui précède, c'est-à-dire aussi bien au niveau de l'onde de choc que du phénomène d'amortissement, il apparait très clairement que les pieux de diamètre 16 , 12 et $10 \mathrm{~mm}$ présentent un comportement similaire, et qu'ils peuvent donc tous les trois simuler le même pieuprototype. La conclusion est un peu plus nuancée pour le pieu de diamètre 8.

TABLEAUXV Décroissance exponentielle. Exponential decreasing.

\begin{tabular}{c|c|c|c} 
pieu & $\alpha$ & $\beta$ & $\mathrm{r}$ \\
\hline$\phi 16$ & 0,977 & $6,1610^{-2}$ & 0,999 \\
\hline$\phi 12$ & 1,041 & $5,6410^{-2}$ & 0,999 \\
\hline$\phi 10$ & 1,009 & $5,8010^{-2}$ & 0,999 \\
\hline ensemble des 3 pieux & 1,011 & $5,8910^{-2}$ & 0,994 \\
\hline
\end{tabular}

\section{5}

\section{Courbes de battage}

Comme il a été indiqué dans la description du dispositif expérimental, un capteur de déplacement permet de mesurer le mouvement de la tige du vérin, ellemême solidaire de la ventouse par l'intermédiaire du corps du batteur. Les courbes qui ont été enregistrées ne représentent donc pas directement ni le mouvement du marteau, ni le mouvement du pieu. Elles n'en constituent pas moins une "signature» du battage, et à ce titre peuvent être considérées comme de courbes de battage brutes.

On trouvera sur la figure 11 la courbe obtenue pour l'essai 12 a représentative de l'ensemble des courbes obtenues. 


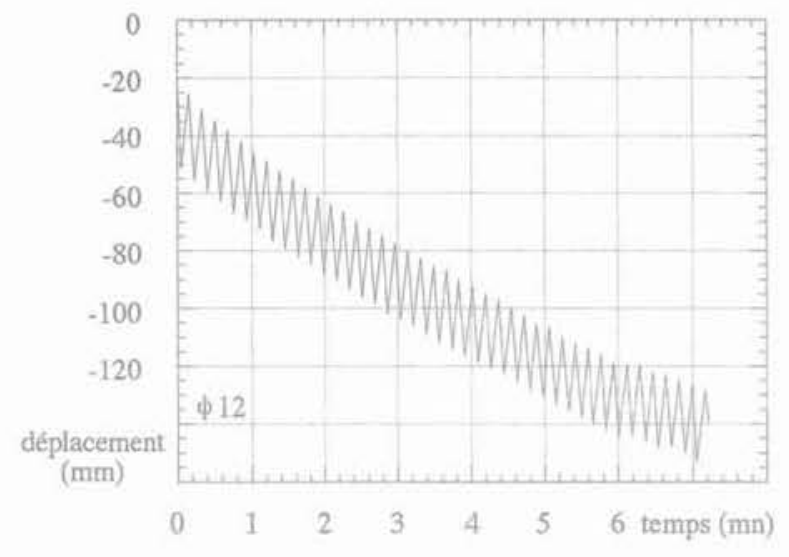

Fด.11 Exemple de courbe de battage brute. Typical recorded driving curve.

Les courbes de battage réelles peuvent être déduites de ces courbes brutes suivant le principe visualisé sur la figure 12

Au départ, le marteau repose sur le pieu, et le batteur est dans une position quelconque, On commence une phase descendante (1) jusqu'à ce que la ventouse soit en contact avec le marteau. Ce point de contact correspond à la fiche initiale du pieu. Après activation de la ventouse, l'ensemble batteur - ventouse - mar-

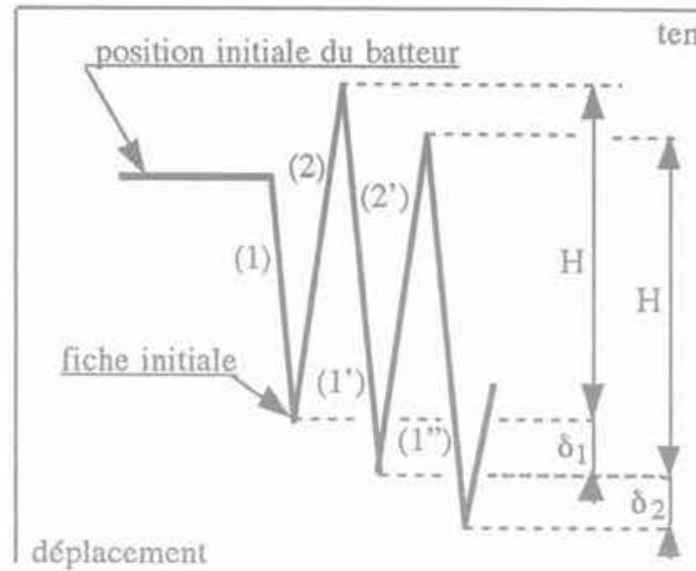

FIG. 12 Principe d'exploitation des courbes brutes.

Driving curves interpretation.

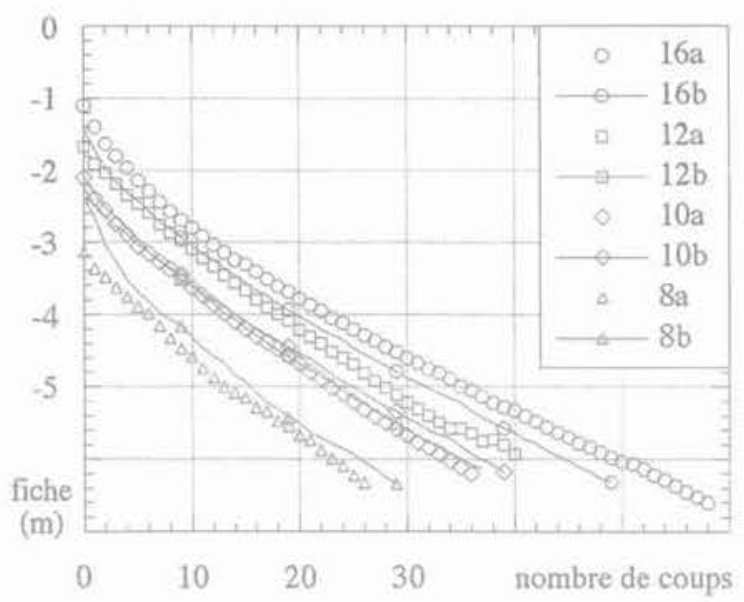

FiG 13 Courbes de battage en données prototypes.

Prototype data driving curves.

teau est remonté (2) de la hauteur H. La ventouse est désactivée, ce qui entraîne la chute du marteau. Une nouvelle phase descendante (1) commence alors jusqu'à ce que la ventouse soit à nouveau en contact avec le marteau, et ainsi de suite.

L'enfoncement $\delta$ du pieu sous chacun des impacts est caractérisé par la différence de niveau de deux positions basses successives. Connaissant la fiche initiale, on peut donc tracer la courbe de battage réelle liant la fiche du pieu au nombre n de coups cumulés. On obtient alors les courbes de la figure 13 .

Sur cette figure, les fiches ont été ramenées au pieuprototype de façon à faciliter la comparaison entre les divers pieux-modèles.

On trouvera dans le tableau XVI les diverses valeurs nécessaires à l'exploitation des courbes brutes.

Dans ce tableau, $\mathrm{f}_{0}$ et $\mathrm{f}_{f}$ césignent respectivement la fiche initiale et la fiche finale $\Delta t$ est l'intervalle de temps moyen entre deux chocs successifs: il est fonction essentiellement des vitesses de montée et de descente du vérin qui sont réglées manuellement. On remarquera que l'on a utilisé une cadence de battage du même ordre de grandeur pour l'ensemble des pieux: on ne satisfait donc pas à la condition de similitude du tableau VII. En fait, l'intervalle de temps entre deux chocs successifs est suffisamment grand pour qu'il ne

TABLEAUXY Caractéristiques du battage. Driving characteristics.

\begin{tabular}{|c|c|c|c|c|c|c|}
\hline $\begin{array}{l}\text { pieu } \\
\text { modèle }\end{array}$ & $\begin{array}{l}\mathrm{f}_{0}(\mathrm{~mm}) \\
\text { modèle }\end{array}$ & $\begin{array}{l}\text { f. (mm) } \\
\text { modèle }\end{array}$ & $\begin{array}{c}f_{0}(m) \\
\text { prototype }\end{array}$ & $\begin{array}{c}f_{1}(m) \\
\text { modèle }\end{array}$ & $n$ & $\Delta t(s)$ \\
\hline $\begin{array}{l}16 a \\
16 b\end{array}$ & $\begin{array}{l}34 \\
44\end{array}$ & $\begin{array}{l}204 \\
195\end{array}$ & $\begin{array}{l}1,10 \\
1,42\end{array}$ & $\begin{array}{l}6,58 \\
6,29\end{array}$ & $\begin{array}{l}58 \\
49\end{array}$ & $\begin{array}{l}11,7 \\
12,6\end{array}$ \\
\hline $\begin{array}{l}12 a \\
12 b\end{array}$ & $\begin{array}{l}40 \\
50\end{array}$ & $\begin{array}{l}142 \\
147\end{array}$ & $\begin{array}{l}1,67 \\
2,08\end{array}$ & $\begin{array}{l}5,92 \\
6,13\end{array}$ & $\begin{array}{l}40 \\
37\end{array}$ & $\begin{array}{r}10,7 \\
9,6\end{array}$ \\
\hline $\begin{array}{l}10 \mathrm{a} \\
10 \mathrm{~b}\end{array}$ & $\begin{array}{l}42 \\
45\end{array}$ & $\begin{array}{l}126 \\
126\end{array}$ & $\begin{array}{l}2,10 \\
2,25\end{array}$ & $\begin{array}{l}6,20 \\
6,20\end{array}$ & $\begin{array}{l}36 \\
39\end{array}$ & $\begin{array}{l}8,9 \\
9,2\end{array}$ \\
\hline $\begin{array}{l}8 a \\
8 b\end{array}$ & $\begin{array}{l}50 \\
38\end{array}$ & $\begin{array}{l}101 \\
101\end{array}$ & $\begin{array}{l}3,12 \\
2,37\end{array}$ & $\begin{array}{l}6,31 \\
6,31\end{array}$ & $\begin{array}{l}27 \\
29\end{array}$ & $\begin{array}{l}10,9 \\
11,4\end{array}$ \\
\hline
\end{tabular}


subsiste plus d'onde de contrainte dans les pieux au moment de l'impact. De ce fait, chaque choc peut être considéré comme isolé, et le non-respect de la condition de similitude doit être sans influence sur les courbes de battage.

On remarquera que les fiches initiales en valeurs prototypes sont très différentes d'un essai à l'autre: en conséquence, il faut éviter de comparer directement les nombres de coups cumulés figurant dans le tableau, mais comparer les nombres de coups cumulés entre deux fiches identiques pour l'ensemble des pieux. C'est ce qui est présenté sur la figure 14 où on a considéré la partie commune aux huit essais, c'est-à-dire entre les fiches 3,1 et $5,9 \mathrm{~m}$.

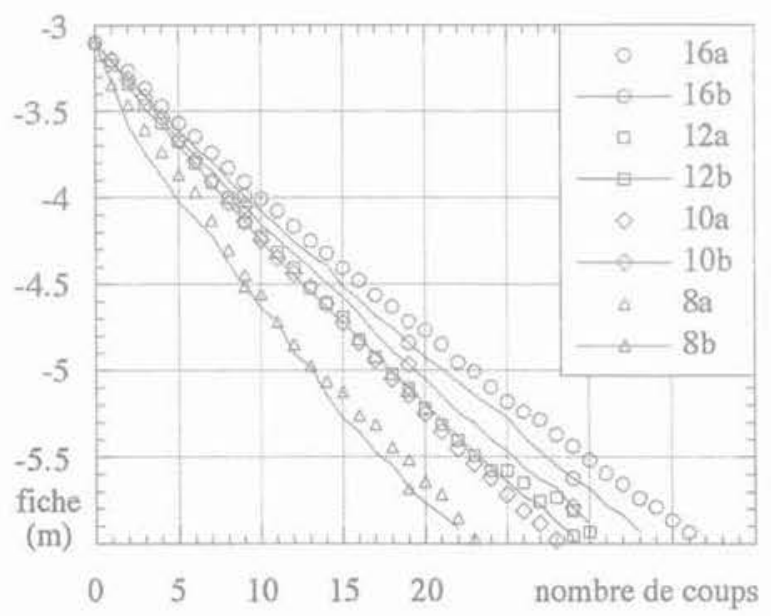

FG. 14 Courbes de battage entre 3,1 et $5,9 \mathrm{~m}$. Plotted driving curves for an embedded depth from 3.1 to $5.9 \mathrm{~m}$.

On constate que globalement, le nombre de coups augmente lorsque le diamètre du pieu-modèle augmente. Or on avait vu précédemment (Tableau XIV) que l'énergie transmise par coup en valeur prototype augmente quand le diamètre du pieu-modèle diminue. On a donc calculé l'énergie transmise $\mathrm{E}_{\mathrm{p}}$ en valeur prototype entre les fiches 3,1 et $5,9 \mathrm{~m}$. Pour cela, on a admis que pour chaque pieu l'énergie transmise par coup (valeurs du tableau XIV] reste constante pendant le battage. Le nombre $n$ de coups nécessaire pour aller de la fiche 3.1 à la fiche 5,9 a été obtenu par interpolation car aucune de ces fiches ne correspond exactement à un impact. Les résultats sont rassemblés dans le tableau XVII.

Ces mêmes résultats sont visualisés sur la figure 15 . Il apparaît clairement que l'énergie nécessaire au fonçage croît lorsque le diamètre du pieu-modèle augmente, et que par conséquent, le facteur d'échelle qui devrait être de $\mathrm{N}^{-3} \mathrm{n}^{\prime}$ est pas respecté.

TABLEAU XVII Énergies transmises entre 3,1 et $5,9 \mathrm{~m}$. Transmitted energies for an embedded depth from 3.1 to $5.9 \mathrm{~m}$.

\begin{tabular}{c|c|c}
\hline pieu & $n$ & $E_{p}(M J)$ \\
\hline $16 a$ & 35,5 & 1,39 \\
\hline $12 a$ & 29,8 & 1,22 \\
\hline $10 a$ & 27,2 & 1,22 \\
\hline $8 b$ & 21,9 & 1,05 \\
\hline
\end{tabular}

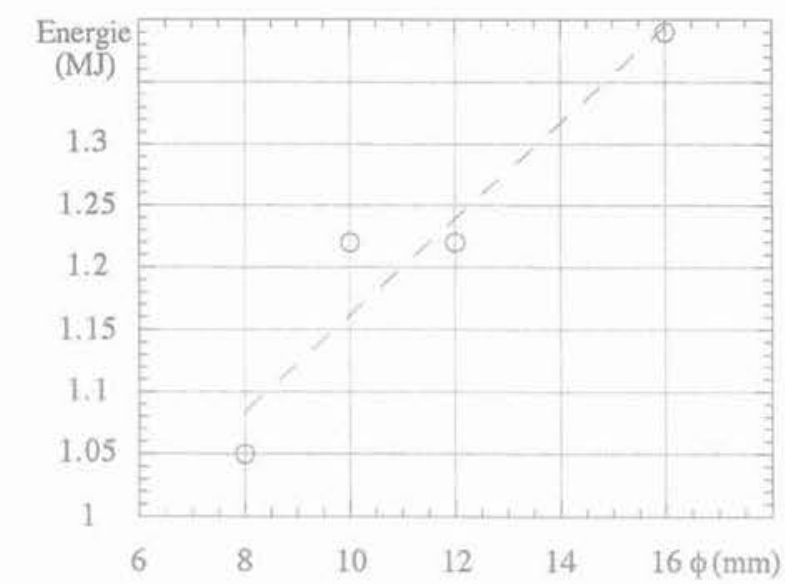

FG 15 Énergies transmises entre 3,1 et 5,9 mètres.

Transmitted energies for an embedded depth from 3.1 to $5.9 \mathrm{~m}$.

\section{Capacité portante}

A l'issue de chaque mise en fiche par battage, il a été procédé à un essai de capacité portante. Il est absolument indispensable de comparer le comportement des pieux-modèles en fonction du facteur d'échelle utilisé. L'une des comparaisons possibles porte sur la capacité portante statique en données prototypes. De façon à respecter le facteur d'échelle des vitesses, la même vitesse d'application du chargement a été utilisée pour l'ensemble des pieux. On a par ailleurs réalisé un essal de capacité portante (à $50 \mathrm{~g}$ ) sur un pieumodèle (M10) moulé (à $1 \mathrm{~g}$ ) de $10 \mathrm{~mm}$ de diamètre.

On trouvera sur la figure 16 en données prototypes les résultats obtenus pour les huit pieux battus en cours de centrifugation, ainsi que pour le pieu moulé.

Les courbes effort - déplacement sont contenues dans un faisceau suffisamment serré et sans ordonnancement relatif pour que l'on puisse affirmer qu'elles ne dépendent pas du facteur d'échelle.

La détermination de la capacité portante $Q$ peut se faire à partir de l'intersection des deux droites du tracé ( asymptote-pente à l'origine $),$ On a reporté dans le

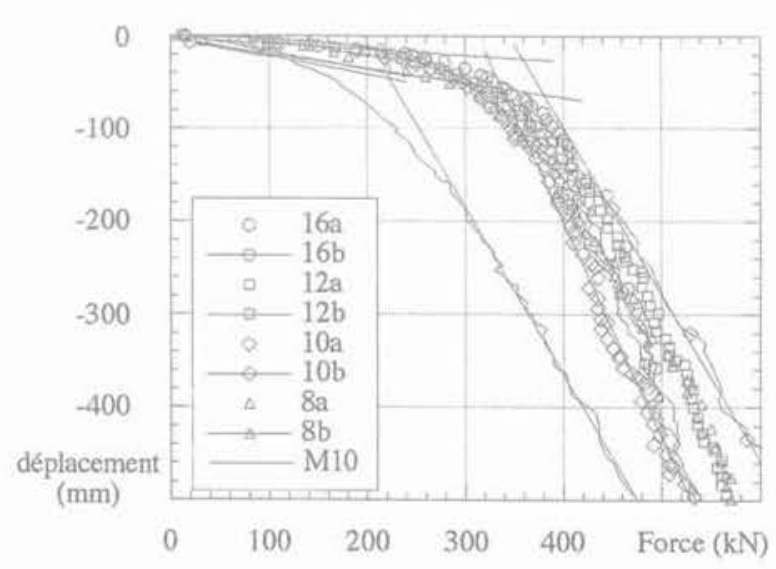

FIG. 16 Essais de chargement en données prototypes.

Prototype data bearing capacity tests. 
TABLEAU XVIII. Capacités portantes en données prototypes. Prototype data bearing capacities.

\begin{tabular}{c|c|c|c|c|c|c|c|c|c} 
Pieu & $16 \mathrm{a}$ & $16 \mathrm{~b}$ & $12 \mathrm{a}$ & $12 \mathrm{~b}$ & $10 \mathrm{a}$ & $10 \mathrm{~b}$ & $8 \mathrm{a}$ & $8 \mathrm{~b}$ & M10 \\
\hline $\mathrm{Q}(\mathrm{kN})$ & 345 & 353 & 383 & 346 & 325 & 365 & 337 & 379 & 224 \\
\hline
\end{tabular}

tableau XVIII les valeurs obtenues pour les huit pieux testés traités indépendamment, ainsi que pour le pieu moulé.

On voit clairement qu'il n'y a pas de relation directe entre la valeur de la capacité portante et le diamètre des pieux-modèles battus en cours de centrifugation. Les fiches finales (Tableau XVI) ne semblent pas non plus influer directement les valeurs de Q. Les écarts (environ $12 \%$ ) entre deux essais semblables se situent dans le domaine habituel de ce type de mesure.

On comparera avec intérêt la valeur de la capacité portante pour les pieux battus:

$$
Q_{b}=(354 \pm 29) \mathrm{kN}
$$

avec celle obtenue pour le pieu foré:

$$
Q_{\mathrm{f}}=224 \mathrm{kN}
$$

L'ensemble de ces résultats est montrẻ sur la figure 17.

On vérifie donc bien ainsi la différence de comportement du pieu en fonction de son mode de mise en œuvre, l'écart sur la capacité portante entre les pieux battus et le pieu moulé étant de plus de $35 \%$.

On aurait pu procéder à une autre forme de dépouillement des essais de capacité portante en

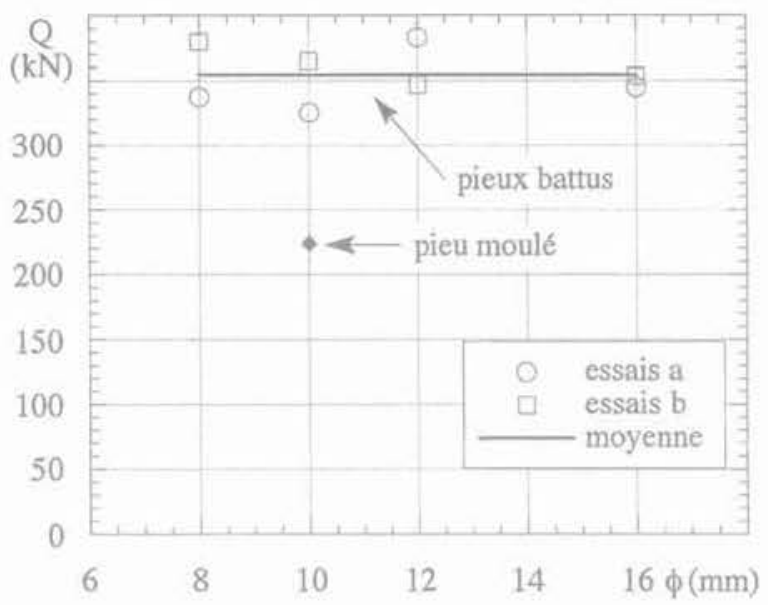

FIG.17 Capacités portantes en données prototypes.

Prototype data bearing capacities. exploitant globalement l'ensemble des essais (voir Fig. 16) en traçant les deux asymptotes et les deux pentes à l'origine extrêmes. Avec ce type d'interprétation on obtient:

$$
Q_{b}=(352 \pm 28) \mathrm{kN}
$$

ce qui correspond à un résultat quasiment identique à l'interprétation essai par essai, et confirme la cohérence des mesures.

5

\section{Conclusion}

Les deux séries d'essais qui ont été réalisées à Bochum et à Nantes dans des conditions très différentes ont permis de justifier pour l'essentiel la validité des lois de similitude dans le cas du battage en cours de centrifugation. On a en particulier vérifié que la capacité portante en données prototypes est indépendante du diamètre du pieu, ce qui était en fait l'objectif principal de la deuxième vérification expérimentale. En revanche, l'énergie transmise est sensible au facteur d'échelle: nous ne saurions pas recommander dans l'état actuel de déduire l'énergie nécessaire pour foncer un pieu-prototype à partir de l'énergie mesurée sur un pieu-modèle. Il est certain que l'énergie n'est pas un paramètre suffisant en lui-même, et que la forme de l'onde de contrainte qui dépend directement de la qualité du contact entre le marteau et le pieu - intervient dans la loi de l'interaction entre le sol et le pieu. Par ailleurs, la manipulation de pieux de petits diamètres s'avère délicate et nécessite une très grande précision pour tous les paramètres. II semble quavec le pieu de diamètre $8 \mathrm{~mm}$ la limite était pratiquement atteinte compte tenu du matériel utilisé. Enfin, il a été vérifié de façon tout à fait incontestable que le mode de mise en place du pieu-modèle doit être identique à celui du pieu-prototype simulé.

\section{Remerciements}

Les auteurs remercient pour leur soutien financier: - les autorités franco-allemandes responsables du programme PROCOPE «Dynamique appliquée au Génie Civil n:

- le MESR et le METT responsables du programme "Macrogravité $92 / 93$.

\section{Bibliographie}

[1] Levacher D. - Modélisation numérique et physique des pieux et simulation en centrifugeuse du comportement des pieux. Thèse de Doctorat d'État, École Centrale de Nantes - Université de Nantes, janvier 1992, 189p.

[2] Bouafia A., Garnier J., Levacher D. Comportement d'un pieu isolé chargé latéralement dans le sable. Colloque Fondations Profondes, mars 1991, Paris, Presses de l'ENPC, pp. 129-137.
[3] Thiel G., Sieffert J.-G. - Primär-Ergebnisse der Modellversuche zur dynamischen Pfahilbelastung an der geotechnischen Großzentrifuge. Programme PROCOPE. ENSM Nantes - RU Bochum, Rapport d'essais, nov. 1988, 166 p.

[6] Thiel G., Sieffert J,-G. - Essais de battage en cours de centrifugation. Application à la capacité portante des pieux. Programme PROCOPE. ENSM Nantes - RU Bochum, Rapport final 1987-1989, pp. 27-35.
[5] Levacher D., Sieffert J.-G. Cottineau L.. M. - Le battage des pieux sur modèles centrifugés. Séminaire de bilan. Programme Macrogravitè 92/93, Nantes, mars 1994, pp. 24-32.

[6] Sieffert J.G., Levacher D. - Etude des conditions de similitude dans le cas du battage des pjeux. Rapport final du Programme Macrogravité 92/93, juillet $1994,45 p$. 